\title{
Exchange of thiol-disulfide pairs in scorpion envenomation
}

\author{
Merve Ergin Tuncay', (1) Betul Ozbek Iptec', (1) Ozlem Bingol'2, () Mustafa Caliskanturk³, \\ Salim Neselioglu', , (D) Ozcan Erel' ${ }^{1}$
}

'Department of Biochemistry, Yildirim Beyazit University, Faculty of Medicine, Ankara, Turkey

${ }^{2}$ Department of Emergency Medicine, Soke Fehime Faik Kocagoz State Hospital, Aydin, Turkey

${ }^{3}$ Department of Emergency Medicine, 25 Aralik State Hospital, Gaziantep, Turkey

\begin{abstract}
Objectives: The present study aims to analyze thiol-disulfide profile tests in scorpion envenomation. Methods: This study included 35 patients with scorpion envenomation and 41 healthy individuals. Thiol-disulfide test panel and myeloperoxidase and catalase activities were determined in both groups.

Results: Patients with scorpion envenomation group had significantly higher native thiol concentrations and significantly lower disulfide amounts than the control group ( $p=0.001$, for both). Also, total thiol levels were higher in patients than healthy individuals $(p>0.05)$. Significantly decreased the disulfide/native thiol ratios and significantly increased disulfide/total thiol ratios and native/total thiol ratios were obtained in patients with scorpion sting than in the healthy subjects ( $p<0.001$, for all ratios). Both catalase and myeloperoxidase activities increased in patients with scorpionism than controls $(p<0.05$, for both).There were powerful relationships among enhanced myeloperoxidase activities and thiol-disulfide system tests $(\mathrm{p}<0.05$, for all).

Conclusion: The equilibrium between thiol-disulfide couples was disrupted in scorpion envenomation. As thiol metabolism is a key component in inflammatory, immune and detoxification mechanisms, excessive thiols may be a response to these processes in scorpionism.
\end{abstract}

Keywords: Antioxidant, disulfide, oxidative stress, scorpion envenomation, thiol; thiol-disulfide homeostasis

$S_{l e m}^{c o n}$ corpion envenomation (SE) is a widespread health problem in some parts of the world. SE is a substantial cause of morbidity and mortality. SE presents a broad range of clinic manifestations [1]. Scorpionism develops local symptoms, mostly. Severe SE leads to hyperactivation of the autonomic nervous system due to the direct effect of venom toxins resulting in cardiovascular, nervous, respiratory and gastrointestinal system dysfunctions [2]. The pathogenesis of scorpion envenomation comprises a complicated response of cellular activation; oxidative stress and inflammation are two critical and central elements implicated in the venom-induced tissue injury $[3,4]$.

Various molecules have been determined in scorpion venom like mucopolysaccharides, lipids and peptides [5]. Scorpion venom consists of two groups of peptides, disulfide-bridged peptides and non-disulfide-bridged peptides [6]. Disulfidebridged peptides deteriorate the functions of ion channels [7]. Hence, they are recognized as the most powerful toxins in scorpion venom [5]. Non-disulfide-bridged peptides exhibit hemolytic and immune-regulatory activities [5]. The effects of scorpion envenomation are essentially mediated via the manner of neuropeptides on several ion channels in membranes [7]. Ion channels display a vital role in all sides of cellular functions. Post-translational modifications are significant mechanisms modulating ion channel functions [8]. Thiols $(-\mathrm{S}-\mathrm{H})$ are target for the redox related post-translational modifications. Redox related post-translational modifications are present in both physiological and pathological situations involving ox-

Address for correspondence: Merve Ergin Tuncay, MD. Department of Biochemistry, Yildirim Beyazit University, Faculty of Medicine, Ankara, Turkey Phone: +90 5532534234 E-mail: erginmerve@hotmail.com ORCID: 0000-0003-3440-3353

Submitted Date: May 28, 2020 Accepted Date: June 28, 2020 Available Online Date: October 05, 2020

${ }^{\circ}$ Copyright 2020 by International Journal of Medical Biochemistry - Available online at www.internationalbiochemistry.com

OPEN ACCESS This work is licensed under a Creative Commons Attribution-NonCommercial 4.0 International License. 
idative stress [9]. Thiol groups are vulnerable to oxidation [8]. In oxidative situation, thiols form a disulfide bond in response to the redox environment [10]. Formed disulfide bonds can be turned into thiol groups again [10]. Dynamic thiol-disulfide homeostasis has been involved in a wide range of processes, such as antioxidant defense, detoxification, redox signaling and signal transduction mechanisms [10].

The alterations in the thiol-disulfide network have been discussed in several disease conditions [11-14]. The present study aims to determine the thiol-disulfide balance in scorpion envenomation. To our knowledge, this study compromises the first report in this area.

\section{Materials and Methods}

This prospective study comprised 35 patients who were admitted with scorpion sting to the emergency department. Diagnosis of scorpion envenomation depended on the patients' history, clinical features and laboratory findings. Scorpion sting time was gained from the patient's history. The applicants to the hospital within 24 hours after the scorpion sting were included in this study. Patients suffering from any systemic, autoimmune, inflammatory and infectious diseases were excluded from this study. Moreover, patients who were receiving medications or supplements were not enrolled in this study. The control group consisted of 41 healthy subjects with normal physical examination and routine clinical laboratory test results. Patients and healthy individuals were matched concerning age and sex. The study procedure was approved by the local ethics committee. All subjects in the study group were informed about this study and their consents were taken.

Venous blood samples were obtained from all participants by venipuncture on arrival. The serum was then separated from the cell by centrifugation at $1700 \mathrm{~g}$ for $10 \mathrm{~min}$ and stored at $-80^{\circ} \mathrm{C}$ until analysis was performed. Myeloperoxidase activity was determined by a modification of the o-dianisidine method [15]. Catalase activity was determined by the method described by Goth [16].

Thiol-disulfide tests were quantified using the most recently defined spectrophotometric assay. In this method, the reductant sodium borohydride reduced the disulfide forms (-S-S) to free thiols. Afterwards, the pre-existing native thiols and reduced free thiols were determined using 5, 5'-dithiobis-(2nitrobenzoic) acid (DTNB). The obtained measurement shows the amount of total thiols (-S-H+-S-S). The amount of disulfide is found from the equation, which is half of the difference of native $(-\mathrm{S}-\mathrm{H})$ and total thiol levels. Related proportions disulfide/native thiol $(-\mathrm{S}-\mathrm{S} /-\mathrm{S}-\mathrm{H})$, disulfide/total thiol $[-\mathrm{S}-\mathrm{S} /$ $(-\mathrm{S}-\mathrm{H}+-\mathrm{S}-\mathrm{S})]$, and native thiol/total thiol $[-\mathrm{S}-\mathrm{H} /(-\mathrm{S}-\mathrm{H}+-$ $\mathrm{S}-\mathrm{S})$ ] were calculated from the obtained amounts of disulfide bonds, native and total thiols.

Statistical analyses were conducted using SPSS software version 22 (SPSS Inc. Chicago, IL). Data distribution was evaluated using visual and statistical methods. Because the variables exhibited normal distribution, independent sample t-tests were performed to compare the parameters among groups. To assess the relationships between parameters, Pearson's correlation analysis was applied. In all analyses, the statistical significance was assigned as a p-value of less than 0.05 .

\section{Results}

Demographic data and laboratory findings are indicated in Table 1. No statistically significant differences were observed concerning age and sex. There were no significant differences between laboratory tests except glucose. In patients, two subjects had no symptoms, 18 subjects had pain, eight subjects had hyperemia, one had edema, four subjects had nausea, and two subjects had both nausea and vomiting. All patients were treated symptomatically. Only 11 of the patients received antivenom. All patients were conscious on admission to the emergency department. None of the patients were taken into intensive care. Moreover, no mortality occurred.

Outcomes concerning thiol-disulfide tests and certain antioxidant variables are demonstrated in Table 2. In the scorpion envenomation group, native thiol levels were significantly higher than in the control group $(p=0.001)$. Although total thiol levels were higher in the scorpion envenomation group than in the healthy controls, the difference between mean total thiol levels was not statistically significant in the two groups ( $p>0.05)$. When the two groups were assessed, based upon disulfide amounts, there was a significant difference between the groups $(p=0.001)$, and disulfide amounts were lower in the scorpion envenomation patients than in the healthy subjects. Furthermore, the disulfide/native thiol ratios and disulfide/total thiol ratios were significantly lower, and native/total thiol ratios were significantly higher in patients with scorpion sting than in the healthy individuals $(p<0.001$, for all ratios).

Myeloperoxidase and catalase activities significantly increased in patients with the scorpion envenomation compared with the control group (Table 2). When the relationships between the thiol-disulfide profile tests and antioxidant enzymes were examined, there were positive correlations between myeloperoxidase activities and native thiol levels and native/total thiol ratios $(r=0.33, p<0.05$ and $r=0.47, p<0.001$ respectively). In addition, myeloperoxidase activities significantly and negatively correlated with disulfide amounts and the disulfide/native thiol and disulfide/total thiol ratios $(r=-0.27, p<0.05 ; r=-0.47, p<0.001$ and $r=-0.47$, $p<0.001$, respectively).

\section{Discussion}

Scorpion envenomation is a prevalent health hazard and recognized as a substantial risk element for morbidity and mortality [2]. However, most stings result in only local effects; sys- 
Table 1. Clinical characteristics and certain laboratory findings of the study population

\begin{tabular}{|c|c|c|c|}
\hline & Scorpion envenomation $(n=35)$ & Control group $(n=41)$ & $\mathbf{p}$ \\
\hline Gender (male/female) & $20 / 15$ & $25 / 16$ & NS \\
\hline Total protein (g/dl) & $7.28 \pm 0.46$ & $7.57 \pm 0.33$ & NS \\
\hline Glucose (mg/dl) & $96.11 \pm 8.5$ & $87.92 \pm 7.77$ & $\mathrm{p}<0.05$ \\
\hline AST (U/L) & $21.22 \pm 4.14$ & $19.38 \pm 6.46$ & NS \\
\hline ALT (U/L) & $25.6 \pm 4.53$ & $24.07 \pm 6.12$ & NS \\
\hline Sodium (mmol/L) & $139.9 \pm 2.33$ & $141 \pm 1.24$ & NS \\
\hline Potassium (mmol/L) & $4.39 \pm 0.35$ & $4.52 \pm 0.27$ & NS \\
\hline Chloride (mmol/L) & $102 \pm 1.88$ & $103 \pm 2.06$ & NS \\
\hline PT (second) & $13.7 \pm 1.75$ & $11.83 \pm 1.49$ & NS \\
\hline WBC (x109/L) & $7.9 \pm 1.42$ & $7.60 \pm 1.34$ & NS \\
\hline $\mathrm{HGB}(\mathrm{g} / \mathrm{dl})$ & $13.93 \pm 2.02$ & $14.13 \pm 1.71$ & NS \\
\hline Platelet $\left(\times 10^{9} / \mathrm{L}\right)$ & $241.9 \pm 33.06$ & $248.5 \pm 30.67$ & NS \\
\hline
\end{tabular}

Values are mean \pm SD. $p<0.05$ was accepted as statistically significant. *NS: Non-significant, AST: Aspartate aminotransferase, ALT: Alanine aminotransferase, cTn I: Cardiac troponin I, CK MB: MB fraction of creatine kinase, INR: International normalized ratio, aPTT: Activatedpartial thromboplastin time, PT: Prothrombin time, WBC: White blood cells, HGB: Hemoglobin

Table 2. Thiol-disulfide profiles and certain antioxidant variables of the study group

\begin{tabular}{llll}
\hline Parameters & Scorpion envenomation $(\mathbf{n = 3 5 )}$ & Control group (n=41) & p* \\
\hline Native thiol, $\mu \mathrm{mol} / \mathrm{L}$ & $394.86 \pm 35.53$ & $368.28 \pm 34.75$ & $\mathrm{p}=0.001$ \\
Total thiol, $\mu \mathrm{mol} / \mathrm{L}$ & $424.34 \pm 44.74$ & $408.52 \pm 42.73$ & $\mathrm{p}>0.05$ \\
Disulfide, $\mu \mathrm{mol} / \mathrm{L}$ & $14.74 \pm 6.97$ & $20.11 \pm 6.51$ & $\mathrm{p}=0.001$ \\
Disulfide/native thiol ratio, $\%$ & $3.68 \pm 1.58$ & $5.43 \pm 1.60$ & $\mathrm{p}<0.001$ \\
Disulfide/total thiol ratio, $\%$ & $3.39 \pm 1.37$ & $4.86 \pm 1.37$ & $\mathrm{p}<0.001$ \\
Native thiol/total thiol ratio, $\%$ & $93.21 \pm 2.74$ & $90.27 \pm 2.64$ & $\mathrm{p}<0.001$ \\
Myeloperoxidase, $\mathrm{U} / \mathrm{L}$ & $182.39 \pm 21.35$ & $101.58 \pm 16.31$ & $\mathrm{p}<0.001$ \\
Catalase, $\mathrm{kU} / \mathrm{L}$ & $169.13 \pm 24.88$ & $157.5 \pm 8.58$ & $\mathrm{p}<0.05$ \\
\hline
\end{tabular}

Results were expressed as mean \pm SD. ${ }^{*} \mathrm{p}<0.05$ considered significant

temic effects are considered to be the result of nervous system activation [1]. It can be a life-threating situation particularly in more vulnerable subjects like children and the elderly unless treated appropriately and quickly [17]. Potential complications involving cardiovascular effects, neuromuscular excitation, respiratory failure, hypotension and multi-organ dysfunction may lead to death [2]. Moreover, scorpion envenomation can be categorized as mild, moderate and severe according to the signs and symptoms presented by the patient [18].

Thiol groups are vulnerable to oxidative modification [9]. Thiols could be moderated in answer to exogenous stimulus or alterations in the redox environment [9]. Thiol modifications are thought to be the essential mechanism that modulates the ion channel functions [8]. In addition, a stimulus, such as mechanical or others, may change ion channel activities causing signaling cascades that generate reactive oxygen species or other substances that regulate ion channels [8]. A balance among these modifications is needed for cellular homeostasis and functions $[8,9]$. Hence, alterations in the thiol-disulfide network system involve in various pathways, such as detoxification, inflammation, immune response and antioxidant defense [10]. That the thiol-disulfide redox system takes part in many mechanisms makes it mentioned in many diseases $[10,19]$.

The mechanism behind the toxicity of scorpion envenomation is not clearly understood [4]. The pathogenesis of the toxic effects of scorpion venom is noticeably complicated 
[4]. Toxins target ion channels and delay ion channels inactivation [7]. Despite that, this cannot explain the necrosis in multiple organs. Scorpion venom is thought to enhance free radical generation, which may trigger the onset of tissue damage [3]. Additionally, it has been demonstrated that scorpion toxins activate excessive immune and inflammatory response [20]. To our knowledge, no prior study investigating thiol-disulfide homeostasis in scorpion envenomation has been published.

As presented in Table 2, native and total thiol levels were higher, and disulfide amounts were lower in patients with scorpion envenomation than controls. These findings implicate that there was an overproduction of thiols and the reduction of disulfides in scorpion envenomation and also, the oxidized side of the thiol-disulfide system shifted toward to the reduced side. Thiols may have increased as they are involved in the immune system and detoxification. Moreover, significantly increased native/total thiol ratios and diminished disulfide/native thiol ratios and disulfide/total thiol ratios pointed out that the thiol-disulfide balance was disrupted in scorpion envenomation. In addition, myeloperoxidase and catalase activities were significantly higher in the scorpion envenomation group than the control group. These results were an indicator of the augmented antioxidant defense system in scorpionism. Significant relationships among thiol-disulfide profile tests and myeloperoxidase activities have reinforced the obtained outcomes.

Markers of the antioxidant-oxidant system have been evaluated in some studies $[17,21]$. Al et al. assessed the total antioxidant and oxidant status [21]. They found antioxidant and oxidant status levels higher in scorpion envenomed patients than controls [21]. The elevation of total antioxidant status is come in concordance with increased thiols and antioxidant enzyme activities in our study.

Scorpion venoms are recognized to enhance the excessive inflammatory responses [4]. The inflammatory response was featured via the activation of macrophages and neutrophils. A broad range of studies revealed the induced cytokines and anti-inflammatory molecules production, complement activation and NO generation during scorpionism [4]. Myeloperoxidase, in neutrophils, exists in the antioxidant defense system, is released during inflammatory conditions [14]. It has been experimentally demonstrated that increased myeloperoxidase levels were found in the lung of animals that had received venom than controls [22].

$\mathrm{N}$-acetylcysteine (NAC) displays potent antioxidant and antiinflammatory effects due to the sulfhydryl group in its configuration. NAC is a substantial precursor in the generation of glutathione [23]. On the other hand, NAC prohibits tissue damage by stimulating the glutathione levels. Hence, it could be a component of the detoxification response in the liver [23]. Dousset et al. examined the protective effects of antioxidants during scorpion envenomation [20]. They have observed that prior treatment of NAC decreased the toxicity [20].

\section{Conclusion}

In conclusion, there is an imbalance between thiol-disulfide couples in scorpion envenomation. Enhanced native and total thiol levels represent a strengthened antioxidant defense system in scorpion envenomation. Powerful associations between increased myeloperoxidase activities and thiol-disulfide tests support this phrase. Because the thiol-disulfide network system is a component of both the detoxification mechanism and inflammatory and immune response, future studies may provide novel therapeutic approaches for scorpionism. Not knowing the thiol-disulfide levels and other parameters levels of the patients before the scorpion sting (basal) is a limitation of this study.

Conflict of Interest: No confict of interest.

Ethics Committee Approval: This study was approved by Ankara City Hospital Clinical Researches Ethics Committee (Date: 07.05.2020 and No: 508).

Financial Disclosure: None declared.

Peer-review: Externally peer-reviewed.

Authorship Contributions: Concept - M.E.T.; Design - M.E.T., O.B., M.C.; Supervision - M.E.T., O.B., M.C., B.O.I., S.N., O.E.; Funding - O.E.; Materials - M.E.T., O.B., M.C.; Data collection \&/or processing - M.E.T., O.B., M.C., B.O.I.; Analysis and/or interpretation M.E.T., O.B., M.C., B.O.I., S.N., O.E.; Literature search - M.E.T.; Writing - M.E.T.; Critical review - M.E.T., O.B., M.C., B.O.I., S.N., O.E.

\section{References}

1. Santos MS, Silva CG, Neto BS, Grangeiro Júnior CR, Lopes VH, Teixeira Júnior AG, et al. Clinical and Epidemiological Aspects of Scorpionism in the World: A Systematic Review. Wilderness Environ Med 2016;27(4):504-18. [CrossRef]

2. Isbister GK, Bawaskar HS. Scorpion envenomation. N Engl J Med 2014;371(5):457-63. [CrossRef]

3. Khemili D, Laraba-Djebari F, Hammoudi-Triki D. Involvement of Toll-like Receptor 4 in Neutrophil-Mediated Inflammation, Oxidative Stress and Tissue Damage Induced by Scorpion Venom. Inflammation 2020;43(1):155-67. [CrossRef]

4. Reis MB, Zoccal KF, Gardinassi LG, Faccioli LH. Scorpion envenomation and inflammation: Beyond neurotoxic effects. Toxicon 2019;167:174-9. [CrossRef]

5. Alirahimi E, Kazemi-Lomedasht F, Shahbazzadeh D, Habibi-Anbouhi M, Hosseininejad Chafi M, Sotoudeh N, et al. Nanobodies as novel therapeutic agents in envenomation. Biochim Biophys Acta Gen Subj 2018;1862(12):2955-65. [CrossRef]

6. Zeng XC, Corzo G, Hahin R. Scorpion venom peptides without disulfide bridges. IUBMB Life 2005;57(1):13-21. [CrossRef]

7. Housley DM, Housley GD, Liddell MJ, Jennings EA. Scorpion toxin peptide action at the ion channel subunit level. Neuropharmacology 2017;127:46-78. [CrossRef]

8. Yang $Y$, Jin $X$, Jiang C. S-glutathionylation of ion channels: insights into the regulation of channel functions, thiol modifi- 
cation crosstalk, and mechanosensing. Antioxid Redox Signal 2014;20(6):937-51. [CrossRef]

9. Moran LK, Gutteridge JM, Quinlan GJ. Thiols in cellular redox signalling and control. Curr Med Chem 2001;8(7):763-72.

10. Erel O, Neselioglu S. A novel and automated assay for thiol/ disulphide homeostasis. Clin Biochem 2014;47(18):326-32.

11. Ergin M, Caliskanturk $M$, Senat A, Akturk O, Erel O. Disulfide stress in carbon monoxide poisoning. Clin Biochem 2016;49(16-17):1243-7. [CrossRef]

12. Sanhal CY, Daglar K, Kara O, Yılmaz ZV, Turkmen GG, Erel O, et al. An alternative method for measuring oxidative stress in intrahepatic cholestasis of pregnancy: thiol/disulphide homeostasis. J Matern Fetal Neonatal Med 2018;31(11):1477-82.

13. Ergin Tuncay M, Erkılıc A, Gunes A, Nural C, Erel O. A remarkable point for evaluating the severity of burns: Thiol-disulfide profile. Burns 2020;46(4):882-7. [CrossRef]

14. Tufan ZK, Hasanoglu I, Kolgelier S, Alisik M, Ergin M, Yilmaz GR, et al. A retrospective controlled study of thiol disulfide homeostasis as a novel marker in Crimean Congo hemorrhagic fever. Redox Rep 2017;22(6):241-5. [CrossRef]

15. Bradley PP, Priebat DA, Christensen RD, Rothstein G. Measurement of cutaneous inflammation: estimation of neutrophil content with an enzyme marker. J Invest Dermatol 1982;78(3):206-9. [CrossRef]

16. Góth L. A simple method for determination of serum catalase activity and revision of reference range. Clin Chim Acta 1991;196(2-3):143-51. [CrossRef]
17. Ahmed AE, Hassan MH, Rashwan NI, Sayed MM, Meki AMA. Myocardial injury induced by scorpion sting envenoming and evidence of oxidative stress in Egyptian children. Toxicon 2018;153:72-7. [CrossRef]

18. Cesaretli Y, Ozkan O. Scorpion stings in Turkey: epidemiological and clinical aspects between the years 1995 and 2004. Rev Inst Med Trop Sao Paulo 2010;52(4):215-20. [CrossRef]

19. Kundi $H$, Ates I, Kiziltunc E, Cetin M, Cicekcioglu H, Neselioglu $\mathrm{S}$, et al. A novel oxidative stress marker in acute myocardial infarction; thiol/disulphide homeostasis. Am J Emerg Med 2015;33(11):1567-71. [CrossRef]

20. Dousset E, Carrega L, Steinberg JG, Clot-Faybesse O, Jouirou $B$, Sauze N, et al. Evidence that free radical generation occurs during scorpion envenomation. Comp Biochem Physiol C Toxicol Pharmacol 2005;140(2):221-6. [CrossRef]

21. Al B, Yarbil P, Zengin S, Taysi S, Örkmez M, Yildirim C et al. Evaluation of Oxidants and Antioxidants in Scorpion Envenomation. Turkiye Klinikleri J Med Sci 2013;33(4):1119-26. [CrossRef]

22. Adi-Bessalem S, Mendil A, Hammoudi-Triki D, Laraba-Djebari F. Lung immunoreactivity and airway inflammation: their assessment after scorpion envenomation. Inflammation 2012;35(2):501-8. [CrossRef]

23. Rushworth GF, Megson IL. Existing and potential therapeutic uses for $\mathrm{N}$-acetylcysteine: the need for conversion to intracellular glutathione for antioxidant benefits. Pharmacol Ther 2014;141(2):150-9. [CrossRef] 